\title{
An Annuitization Problem in the Tax-Deferred Annuity Model
}

\author{
Yanan Li $\mathbb{D}$ \\ School of Finance, Capital University of Economics and Business, Beijing 100070, China \\ Correspondence should be addressed to Yanan Li; 415758824@qq.com
}

Received 7 July 2021; Accepted 2 August 2021; Published 13 August 2021

Academic Editor: Linyi Qian

Copyright (c) 2021 Yanan Li. This is an open access article distributed under the Creative Commons Attribution License, which permits unrestricted use, distribution, and reproduction in any medium, provided the original work is properly cited.

This paper examines the optimal annuitization, investment, and consumption strategies of an individual facing a time-dependent mortality rate in the tax-deferred annuity model and considers both the case when the rate of buying annuities is unrestricted and the case when it is restricted. At the beginning, by using the dynamic programming principle, we obtain the corresponding HJB equation. Since the existence of the tax and the time-dependence of the value function make the corresponding HJB equation hard to solve, firstly, we analyze the problem in a simpler case and use some numerical methods to get the solution and some of its useful properties. Then, by using the obtained properties and Kuhn-Tucker conditions, we discuss the problem in general cases and get the value functions and the optimal annuitization strategies, respectively.

\section{Introduction}

Referring to [1], we know that, with the aging population, the basic endowment insurance has been unable to meet people's insurance needs. In order to guarantee the sustainable operation of the old age insurance system, many governments begin to adopt preferential tax policies and attract people to buy tax-deferred annuities. In the United States, tax-deferred annuities have benefited more than $60 \%$ of families and become the main source of continuous growth of pension assets in the past 30 years. Tax-deferred annuities also reduce the pension burden in many countries such as Germany and France. However, in some countries, the pilot of tax extension type endowment insurance is not successful. Since tax-deferred annuities can not only play a positive role in improving the three pillar endowment insurance system, but can also alleviate social problems caused by the aging population, prosper the commercial endowment insurance market, and provide investment funds for the securities market, the promotion of tax-deferred annuities is extremely essential and urgent in the whole world.

In order to attract more and more people to buy taxdeferred annuities, the government should know how to adopt tax preferential policies, and insurance companies should know how to design and publicize products. In this paper, by using stochastic optimal methods and the Kuhn-Tucker conditions, we get the public's strategies on how to buy tax-deferred annuities. Then, we can give some suggestions for the government and insurance companies to promote tax-deferred annuities.

Up till now, there have been a lot of papers investigating individuals' optimal annuitization strategies in a taxable annuity model. Reference [2] investigates the optimal annuitization strategy for an infinitely lived individual who faces a choice between voluntary annuitization and discretionary management of assets with systematic withdrawal for consumption purposes. Reference [3] examines the optimal annuitization, investment, and consumption strategies of a utility-maximizing retiree facing a stochastic time of death under an all-or-nothing arrangement and an open-market structure arrangement, respectively. Reference [4] solves a problem of finding the optimal time of annuitization for a retiree having the possibility of choosing her investment and consumption strategy. Reference [5] aims at maximizing the expected utility of consumption with commutable life annuities and finds that the optimal annuitization strategy depends on the size of proportional surrender charge. For a future reference, we can refer to $[6-9]$. 
However, there are only a few articles that investigate the tax-deferred annuities. Reference [10] gives some comparisons of different tax regimes applied to private pensions. Reference [11] identifies four potential risks associated with tax-deferred. Reference [12] concerns with the risk of fluctuating tax rates and changing tax brackets; it also examines how the saver's tax credit changes optimal tax-deferral choices of individuals. These papers are all analyzed by using the data processing method, and they are focusing on comparing the tax-deferred annuity and an ordinary one. This paper investigates the optimal annuitization, investment, and consumption strategies for an individual under EET tax payment mode (in which individuals deposit the premium into an independent account, and the insurance company invests it in the financial market and returns the income to the individual in the form of annuities on retirement. Both the taxes of premiums and investment incomes are deferred until receiving annuities) by using stochastic optimal control theory. We consider both the case when the rate of buying annuities is unrestricted and the case when it is restricted. We get the value functions and the optimal annuitization strategies, respectively.

This paper is organized as follows. In Section 2, the mathematical formulation of the problem is presented. In Section 3, we investigate the optimal investment, consumption, and annuitization strategies when the rate of buying annuities is unrestricted. In Section 4, the same problem is considered when the rate of buying annuities is restricted. Finally, the numerical method is provided in Appendix.

\section{Problem Formulation}

Let us consider the financial market first. Suppose that there are a risk-free asset and a risky asset in the financial market. Their prices per share $B(t)$ and $R(t)$ follow the following equations:

$$
\begin{aligned}
& \mathrm{d} B(t)=r B(t) \mathrm{d} t \\
& \mathrm{~d} R(t)=R(t)[\mu \mathrm{d} t+\sigma \mathrm{d} W(t)] .
\end{aligned}
$$

Here, $r>0$ represents the risk-free rate of return, $\mu>r$ is the drift coefficient of the risk asset, $\sigma>0$ is the volatility coefficient of the risk asset, $W(t)$ is a standard Brownian motion on a complete probability space $(\Omega, \mathscr{F}, P)$, and $\mathscr{F}_{t}$ is the natural filtration generated by $W(t)$.

Let $b_{1, t}$ be the wealth of the individual invested in the risky asset, and $p_{t}$ the rate of buying tax-deferred annuities. Setting aside taxes, we can get that the individual's wealth process under these policies denoted by $x_{t}^{b_{1}, p}$ satisfies

$$
\mathrm{d} x_{t}^{b_{1}, p}=\left[r x_{t}^{b_{1}, p}+b_{1, t}(\mu-r)-p_{t}\right] \mathrm{d} t+\sigma b_{1, t} \mathrm{~d} W(t) .
$$

Like a general tax policy, we suppose that there is a threshold $b>0$; if the income rate is less that $b$, nothing will be taxed; otherwise, the part above $b$ will be taxed with a tax rate $k$. Here, we also assume that the income rate is greater than $b$ since, otherwise, there is no tax preference to buy taxdeferred annuities for the individual. Then, the taxed wealth $\mathrm{d} X_{t}^{b_{1}, p}$ satisfies

$$
\begin{aligned}
\mathrm{d} X_{t}^{b_{1}, p}= & {\left[r X_{t}^{b_{1}, p}+b_{1, t}(\mu-r)-p_{t}\right] \mathrm{d} t+\sigma b_{1, t} \mathrm{~d} W(t) } \\
& -k\left[\left(r X_{t}^{b_{1}, p}+b_{1, t}(\mu-r)-p_{t}-b\right) \mathrm{d} t+\sigma b_{1, t} \mathrm{~d} W(t)\right] \\
= & {\left[\widehat{r} X_{t}^{b_{1}, p}+b_{1, t}(\widehat{\mu}-\widehat{r})-\widehat{p}_{t}\right] \mathrm{d} t+\widehat{\sigma} b_{1, t} \mathrm{~d} W(t)+k b \mathrm{~d} t . }
\end{aligned}
$$

Here,

$$
\begin{aligned}
\widehat{r} & =(1-k) r, \\
\widehat{\mu} & =(1-k) \mu, \\
\widehat{\sigma} & =(1-k) \sigma, \\
\widehat{p}_{t} & =(1-k) p_{t} .
\end{aligned}
$$

Taking consumptions into consideration, we have

$$
\begin{aligned}
\mathrm{d} X_{t}^{b_{1}, p, c}= & {\left[\widehat{r} X_{t}^{b_{1}, p, c}+b_{1, t}(\widehat{\mu}-\widehat{r})-\widehat{p}_{t}\right] \mathrm{d} t } \\
& +\widehat{\sigma} b_{1, t} \mathrm{~d} W(t)+\left(k b-c_{t}\right) \mathrm{d} t,
\end{aligned}
$$

where $c_{t}$ is the consumption rate of the individual at time $t$.

In the pension market, we assume that the insurance company evaluates the residual lifetime using exponentially distributed random variables. Denote the hazard rate evaluated by the individual at time $t$ by $\lambda_{t}^{s}$ and the hazard rate evaluated by the insurance company at time $t$ by $\lambda_{t}^{o}$, respectively. The wealth in the pension fund will also be invested in the financial market. The difference is that the income will not be taxed until the retirement time $T$. So, the income process in the pension fund under investment policy $b_{2, t}$ is

$$
\mathrm{d} Y_{t}^{b_{2}}=\left[r Y_{t}^{b_{2}}+b_{2, t}(\mu-r)+p_{t}\right] \mathrm{d} t+\sigma b_{2, t} \mathrm{~d} W(t) .
$$

Suppose that the individual has to pay taxes at a tax rate $k_{2}$ before he receives annuities. Since the insurance company assumes that the hazard rate of the individual is $\lambda_{t}^{o}$, we can get that the annuity received by the individual is

$$
c_{0}=\frac{\left(1-k_{2}\right) Y_{T}}{A}
$$

where $Y_{T}$ is the total pension fund at the retirement time $T$, $A=\int_{T}^{\infty} e^{-\left(\lambda_{u}^{o}+r\right)} \mathrm{d} u$. Then, the individual's expected cumulative discounted pension is

$$
g\left(Y_{T}\right)=\int_{T}^{\infty} e^{-\left(\lambda_{u}^{s}+r\right)} c_{0} \mathrm{~d} u=\frac{B\left(1-k_{2}\right) Y_{T}}{A}
$$

where $B=\int_{T}^{\infty} e^{-\left(\lambda_{u}^{s}+r\right)} \mathrm{d} u$.

In this paper, we assume that the short selling is allowed, and then, $b_{i, t}, i=1,2$ can be negative. Now, let us give the definition of admissible controls. If $\pi=\left(b_{1, t}^{\pi}, b_{2, t}^{\pi}, p_{t}^{\pi}, c_{t}^{\pi}\right)$ satisfies

(i) $b_{i, t}^{\pi}, i=1,2$ are $\mathscr{F}_{t}$ predictable control processes, and $p_{t}^{\pi}, \quad c_{t}^{\pi}$ are nonnegative $\mathscr{F}_{t}$ predictable control processes.

(ii) $\int_{0}^{\infty} c_{t}^{\pi} \mathrm{d} t<\infty, \quad \int_{0}^{\infty}\left(b_{i, t}^{\pi}\right)^{2} \mathrm{~d} t<\infty, i=1,2, \quad \int_{0}^{\infty} p_{t}^{\pi} \mathrm{d} t$ $<\infty$.

Then, we say that $\pi$ is admissible. Denote all the admissible strategies by $\Pi$. 
Next, let us focus on this question: What are the optimal consumption/investment strategies and the best annuitization strategy for the individual to maximize the sum of his expected accumulated discounted consumption before retirement and the expected discounted wealth at retirement?
Suppose that the individual's wealth at time $t$ is $x$ and his money in his pension account at time $t$ is $y$. Denote the objective function under policy $\pi$ by $J^{\pi}(t, x, y)$, and then, we have

$$
J^{\pi}(t, x, y)=E\left[\int_{t}^{T} \Gamma(t, s) c_{s}^{\pi} \mathrm{d} s+\Gamma(t, T)\left(X_{T}^{\pi}+g\left(Y_{T}^{\pi}\right)\right) \mid X_{t}^{\pi}=x, Y_{t}^{\pi}=y\right],
$$

where $\Gamma(t, s)=e^{-\int_{t}^{s}\left(r+\lambda_{u}^{s}\right) \mathrm{d} u}$. Thus, the value function $V(t, x, y)$ is

$$
V(t, x, y)=\sup _{\pi \in \Pi} J^{\pi}(t, x, y) .
$$

In the following, we give some initial condition assumptions.

(i) Assumption 1

$$
\begin{aligned}
& V_{x}(t, 0, y)>1, \\
& V_{x}(t, 0, y) \geq \frac{V_{y}(t, 0, y)}{1-k}, \quad \forall y \geq 0 .
\end{aligned}
$$

(ii) Assumption 2

$$
\frac{V_{y}(t, 0,0)}{1-k}>1 \text {. }
$$

\section{The Optimal Strategies when $p_{t}$ Is Unrestricted}

In this section, we investigate the optimal strategy of an individual when $p_{t}$ is unrestricted. According to 3.4.2 in [13], we can get the following theorem.

Theorem 1. $V(t, x, y)$ satisfies

$$
\begin{gathered}
\min \left\{\left(r+\lambda_{t}^{s}\right) V(t, x, y)-\sup _{b_{1} \geq 0, b_{2} \geq 0}\left[\left(\widehat{r} x+(\widehat{\mu}-\widehat{r}) b_{1}+k b\right) V_{x}(t, x, y)+\frac{\widehat{\sigma}^{2} b_{1}^{2}}{2} V_{x x}(t, x, y)\right.\right. \\
+\sigma \widehat{\sigma} b_{1} b_{2} V_{x y}+\left(r y+b_{2}(\mu-r)\right) V_{y}(t, x, y)+\frac{\sigma^{2} b_{2}^{2}}{2} V_{y y}(t, x, y)-V_{t}(t, x, y), \\
V_{x}(t, x, y)-1,(1-k) V_{x}(t, x, y)-V_{y}(t, x, y)=0, \quad t<T, \\
V(T, x, y)=x+g(y), \\
V(t, 0,0)=0, \quad t \geq 0 .
\end{gathered}
$$

By taking derivatives with respect to $b_{1}, b_{2}$ in (13), we can get the maximum point:

$$
\begin{aligned}
& b_{1}^{*}(t, x, y)=\frac{(\widehat{\mu}-\widehat{r})\left(V_{y y}(t, x, y) V_{x}(t, x, y)-V_{y}(t, x, y) V_{x y}(t, x, y)\right)}{\widehat{\sigma}^{2}\left(V_{x y}^{2}(t, x, y)-V_{x x}(t, x, y) V_{y y}(t, x, y)\right)}, \\
& b_{2}^{*}(t, x, y)=\frac{(\mu-r)\left(V_{x x}(t, x, y) V_{y}(t, x, y)-V_{x}(t, x, y) V_{x y}(t, x, y)\right)}{\sigma^{2}\left(V_{x y}^{2}(t, x, y)-V_{x x}(t, x, y) V_{y y}(t, x, y)\right)} .
\end{aligned}
$$


The following theorem gives us the solution of (13) in the case when $V_{x}(t, x, y)>1$, and $V_{y}(t, x, y)<(1-k) V_{x}$ $(t, x, y)$, and it is essential in solving (13).

Theorem 2. For $V_{x}(t, x, y)>1, \quad V_{y}(t, x, y)<(1-k)$ $V_{x}(t, x, y)$, we have

$$
V(t, x, y)=V_{1}(t, x)+V_{2}(t, y), \quad 0 \leq t \leq T,
$$

where $V_{1}(t, x)$ satisfies (23) and (20), and $V_{2}(t, y)$ satisfies (24) and (21).

Proof. Denote the optimal rate of buying tax-deferred annuities by $p_{t}^{*}$ and the optimal consumption rate by $c_{t}^{*}$. For $V_{x}(t, x, y)>1, V_{y}(t, x, y)<(1-k) V_{x}(t, x, y)$, we can deduce that $c_{t}^{*}=0, p_{t}^{*}=0$. The individual will never buy annuities in this situation, and there is no interaction between $x$ and $y$. This implies that

$$
V(t, x, y)=V_{1}(t, x)+V_{2}(t, y)
$$

where $V_{1}(t, x)$ are the benefits obtained in the financial market, and $V_{2}(t, y)$ are the benefits obtained from the annuity fund. Then, we can obtain that

$$
\begin{aligned}
V_{1}(t, 0) & =0, \quad t \geq 0, \\
V_{1}(T, x) & =x, \quad x \geq 0, \\
V_{2}(t, 0) & =0, \quad t \geq 0, \\
V_{2}(T, y) & =g(y), \quad y \geq 0 .
\end{aligned}
$$

Taking (16) and (17) into (13), we can get

$$
\begin{aligned}
& \left(r+\lambda_{t}^{s}\right) V(t, x, y)-V_{t}(t, x, y)=\widehat{r} x V_{x}(t, x, y)+r y V_{y} \\
& \quad(t, x, y)+k b V_{x}(t, x, y)-\frac{R^{2}}{2}\left(\frac{V_{x}^{2}(t, x, y)}{V_{x x}(t, x, y)}+\frac{V_{y}^{2}(t, x, y)}{V_{y y}(t, x, y)}\right),
\end{aligned}
$$

where $R=((\mu-r) / \sigma)$. Then, the following two differential equations are satisfied immediately:

$$
\begin{aligned}
& -\left(r+\lambda_{t}^{s}\right) V_{1}(t, x)+V_{1 t}(t, x)+\widehat{r} x V_{1 x}(t, x)+k b V_{1 x}(t, x) \\
& -\frac{R^{2} V_{1 x}^{2}(t, x)}{2 V_{1 x x}(t, x)}=0
\end{aligned}
$$

$$
-\left(r+\lambda_{t}^{s}\right) V_{2}(t, y)+V_{2 t}(t, y)+r y V_{2 y}(t, y)-\frac{R^{2} V_{2 y}^{2}(t, y)}{2 V_{2 y y}(t, y)}=0
$$

According to [14], we know that (23) and (20) or (24) and (21) may be solved by using dual methods. However, the items $\left(r+\lambda_{t}^{s}\right) V_{1}(t, x),\left(r+\lambda_{t}^{s}\right) V_{2}(t, y)$ in (23) and (24) combined with the time dependence of $\lambda_{t}^{s}$ make those partial differential equations hard to solve. In this paper, we use the finite difference method to get $V_{1}(t, x)$ and $V_{2}(t, y)$ in

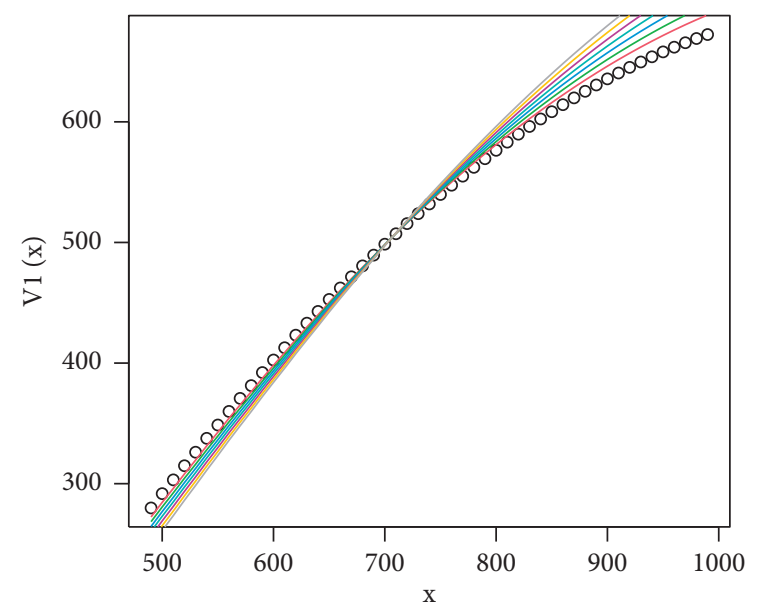

FIGURE 1: $V_{1}(t, x)$

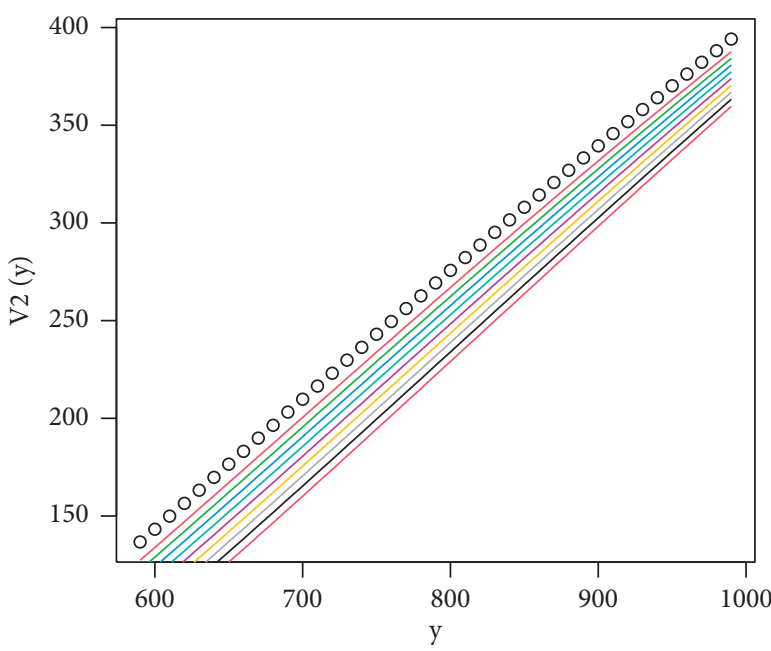

Figure 2: $V_{2}(t, y)$

Appendix A. Considering Figures 1 and 2, we get the following theorem.

Theorem 3. $V_{1}(t, x)$ is a concave increasing positive function with respect to $x, V_{2}(t, y)$ is a concave increasing positive function with respect to $y$. The growth rates of $V_{1}(t, x)$ and $V_{2}(t, y)$ are increasing with respect to $t$.

Next, Let us consider the solutions of (13) in other cases. Firstly, the boundlessness of $p_{t}$ and $c_{t}$ tells us that the individual's optimal consumption and the optimal amount of buying annuities are lump-sum payments. Denote $\Delta_{1}^{*}$ to be the individual's optimal consumption at time $t$, denote $\Delta_{2}^{*}$ to be the optimal amount of buying annuities at time $t$, and considering the right hand of (13), we can obtain that

$$
\begin{gathered}
V(t, x, y)=V\left(t, x-\Delta_{1}^{*}-\Delta_{2}^{*}, y+\frac{\Delta_{2}^{*}}{1-k}\right)+\Delta_{1}^{*}, \\
V_{x}\left(t, x-\Delta_{1}^{*}-\Delta_{2}^{*}, y+\frac{\Delta_{2}^{*}}{1-k}\right)=1,
\end{gathered}
$$




$$
\begin{gathered}
V_{x}\left(t, x-\Delta_{1}^{*}-\Delta_{2}^{*}, y+\frac{\Delta_{2}^{*}}{1-k}\right)=(1-k) \\
\cdot V_{y}\left(t, x-\Delta_{1}^{*}-\Delta_{2}^{*}, y+\frac{\Delta_{2}^{*}}{1-k}\right) .
\end{gathered}
$$

$$
V\left(t, x-\Delta_{1}^{*}-\Delta_{2}^{*}, y+\frac{\Delta_{2}}{1-k}\right)=V_{1}\left(t, x-\Delta_{1}^{*}-\Delta_{2}^{*}\right)+V_{2}\left(t, y+\frac{\Delta_{2}^{*}}{1-k}\right)
$$

Combining it with (25), we can get that

$$
V(t, x, y)=V_{1}\left(t, x-\Delta_{1}^{*}-\Delta_{2}^{*}\right)+V_{2}\left(t, y+\frac{\Delta_{2}^{*}}{1-k}\right)+\Delta_{1}^{*} .
$$

This implies that $V(t, x, y)$ is the solution of the following restricted optimal problem:

$$
P: \sup _{\Delta_{1} \geq 0, \Delta_{2} \geq 0, x-\Delta_{1}-\Delta_{2} \geq 0} V_{1}\left(t, x-\Delta_{1}-\Delta_{2}\right)+V_{2}\left(t, y+\frac{\Delta_{2}}{1-k}\right)+\Delta_{1} .
$$

Here, $\Delta_{1}$ is the individual's consumption strategy, and $\Delta_{2}$ is the wealth of buying annuities. Denote the optimal policy by $\left(\Delta_{1}^{*}(t, x, y), \Delta_{2}^{*}(t, x, y)\right)$.

In order to solve Problem $\mathrm{P}$, we need to do some explicit case analysis. Firstly, define $x_{0}(t), x_{1}(t, y)$ as

$$
\begin{aligned}
& V_{1 x}\left(t, x_{0}(t)\right)=1, \\
& (1-k) V_{1 x}\left(t, x_{1}(t, y)\right)=V_{2 y}(t, y), \quad y \geq 0,
\end{aligned}
$$

Let

$$
\begin{aligned}
& D_{1}=\left\{0 \leq t \leq T, y \geq 0 \mid x_{1}(t, y)<x_{0}(t)\right\} \\
& D_{2}=\left\{0 \leq t \leq T, y \geq 0 \mid x_{1}(t, y) \geq x_{0}(t)\right\}
\end{aligned}
$$

Then, we can get the following two theorems.

Theorem 4. For $(t, y) \in D_{1}$, we have

$$
\begin{aligned}
& \Delta_{1}^{*}(t, x, y)=0, \\
& \Delta_{2}^{*}(t, x, y)= \begin{cases}0, & x \leq x_{1}(t, y), \\
x-x_{1}(t, y), & x>x_{1}(t, y) .\end{cases}
\end{aligned}
$$

Proof. Define

$$
L\left(\Delta_{1}, \Delta_{2}\right)=V_{1}\left(t, x-\Delta_{1}-\Delta_{2}\right)+V_{2}\left(t, y+\frac{\Delta_{2}}{1-k}\right)+\Delta_{1} .
$$

On one hand, we can obtain that

$$
\begin{aligned}
& \frac{\partial L\left(\Delta_{1}, \Delta_{2}\right)}{\partial \Delta_{1}}=1-V_{1}^{\prime}\left(t, x-\Delta_{1}-\Delta_{2}\right), \\
& \frac{\partial L\left(\Delta_{1}, \Delta_{2}\right)}{\partial \Delta_{2}}=\frac{V_{2}^{\prime}\left(t, y+\left(\Delta_{2} /(1-k)\right)\right)}{1-k}-V_{1}^{\prime}\left(t, x-\Delta_{1}-\Delta_{2}\right) .
\end{aligned}
$$

On the other hand, for $(t, y) \in D_{1}$, we have $x_{1}(t, y)<x_{0}(t)$. Then, for $x \leq x_{1}(t, y)<x_{0}(t)$, we can get

$$
\begin{aligned}
V_{1}^{\prime}\left(t, x-\Delta_{1}-\Delta_{2}\right)>V_{1}^{\prime}\left(t, x_{0}(t)\right) & =1, \\
V_{2}^{\prime}\left(t, y+\frac{\Delta_{2}}{1-k}\right) & =(1-k) V_{1}^{\prime}\left(t, x_{1}(t, y)\right) \leq(1-k) V_{1}^{\prime}\left(t, x-\Delta_{1}-\Delta_{2}\right) .
\end{aligned}
$$

That is,

$$
\begin{gathered}
\frac{\partial L\left(\Delta_{1}, \Delta_{2}\right)}{\partial \Delta_{1}}<0, \\
\frac{\partial L\left(\Delta_{1}, \Delta_{2}\right)}{\partial \Delta_{2}} \leq 0 .
\end{gathered}
$$

Clearly, in this case, we have

$$
\begin{aligned}
& \Delta_{1}^{*}(t, x, y)=0, \\
& \Delta_{2}^{*}(t, x, y)=0 .
\end{aligned}
$$

For $x>x_{1}(t, y)$, the signs of $\left(\partial L\left(\Delta_{1}, \Delta_{2}\right) /\right.$ $\left.\partial \Delta_{1}\right)$ and $\left(\partial L\left(\Delta_{1}, \Delta_{2}\right) / \partial \Delta_{2}\right)$ are uncertain. Since

$$
\frac{\partial^{2} L\left(\Delta_{1}, \Delta_{2}\right)}{\partial \Delta_{1}^{2}}=\frac{\partial^{2} L\left(\Delta_{1}, \Delta_{2}\right)}{\partial \Delta_{2}^{2}}=\frac{\partial^{2} L\left(\Delta_{1}, \Delta_{2}\right)}{\partial \Delta_{1} \partial \Delta_{2}}=V_{1}^{\prime \prime}\left(t, x-\Delta_{1}-\Delta_{2}\right) \leq 0
$$

We can get that 


$$
\begin{aligned}
& \frac{\partial^{2} L\left(\Delta_{1}, \Delta_{2}\right)}{\partial \Delta_{1}^{2}}=\frac{\partial^{2} L\left(\Delta_{1}, \Delta_{2}\right)}{\partial \Delta_{2}^{2}} \leq 0 \\
& \frac{\partial^{2} L\left(\Delta_{1}, \Delta_{2}\right)}{\partial \Delta_{1}^{2}} \frac{\partial^{2} L\left(\Delta_{1}, \Delta_{2}\right)}{\partial \Delta_{2}^{2}}-\frac{\partial^{2} L\left(\Delta_{1}, \Delta_{2}\right)}{\partial \Delta_{1} \partial \Delta_{2}}=0 .
\end{aligned}
$$

Then, the Hessian matrix of $L\left(\Delta_{1}, \Delta_{2}\right)$ is seminegative definite, which implies the concavity of $L\left(\Delta_{1}, \Delta_{2}\right)$. So, the necessary and sufficient conditions for the global maximum point are the corresponding Kuhn-Tucker conditions:

$$
\begin{aligned}
& \frac{\partial L\left(\Delta_{1}^{*}(t, x, y), \Delta_{2}^{*}(t, x, y)\right)}{\partial \Delta_{i}} \leq 0, \quad \Delta_{i} \geq 0, \\
& \frac{\partial L\left(\Delta_{1}^{*}(t, x, y), \Delta_{2}^{*}(t, x, y)\right)}{\partial \Delta_{i}} \Delta_{i}=0, \quad i=1,2 .
\end{aligned}
$$

For $\Delta_{1}=0, \Delta_{2}=x-x_{1}(t, y)>0$, we can get

$$
\begin{aligned}
& \frac{\partial L\left(\Delta_{1}, \Delta_{2}\right)}{\partial \Delta_{1}}<0, \\
& \frac{\partial L\left(\Delta_{1}, \Delta_{2}\right)}{\partial \Delta_{2}}=0 .
\end{aligned}
$$

Clearly, the corresponding Kuhn-Tucker conditions are satisfied at $\left(0, x-x_{1}(t, y)\right)$. So, it is the global maximum point.

Theorem 5. For $(t, y) \in D_{2}$, we have

$$
\begin{aligned}
& \Delta_{1}^{*}(t, x, y)= \begin{cases}0, & x \leq x_{0}(t), \\
x-x_{0}(t), & x>x_{0}(t),\end{cases} \\
& \Delta_{2}^{*}(t, x, y)=0 .
\end{aligned}
$$

Proof. The proof is similar to the one in Theorem 4. Now, we omit it.

Remark 1. It is shown from the above two theorems that

(i) In $D_{1}$, the individual will not consume. There is a boundary $x_{1}(t, y)$, for $x>x_{1}(t, y)$, putting the wealth above $x_{1}(t, y)$ to buy annuities is optimal, and for $x \leq x_{1}(t, y)$, putting all the wealth to invest is optimal.

(ii) In $D_{2}$, the individual will not buy annuities. There is a boundary $x_{0}(t)$, for $x>x_{0}(t)$, putting the wealth above $x_{0}(t)$ to consume is optimal, and for $x \leq x_{0}(t)$, putting all the wealth to invest is optimal.

(iii) People of different ages and wealth have different optimal strategies, so, in order to promote tax-deferred annuities, the government should adopt different tax preferential policies for different people, and insurance companies should take different publicity strategies for different people.
Clearly, this is practical, and these theorems give us exact barriers to make decisions.

Remark 2. For $k \geq 1-V_{2 y}(t, y)$, we have

$$
\frac{V_{2 y}(t, y)}{1-k} \geq 1 \text {. }
$$

The concavity of $V_{1}(t, x)$ implies that $x_{1}(t, y) \leq x_{0}(t)$; thus, the individual will not consume, and he will put all the wealth above $x_{1}(t, y)$ to buy annuities. This indicates that raising tax rates properly can stimulate people to buy taxdeferred annuities.

Remark 3. According to Theorem 3, we know that as time goes by, $x_{1}(t, y)$ gets bigger, and the lower bound of purchasing annuities becomes more and more difficult to reach. So, young people are more likely to buy insurance than old ones. Thus, insurance companies should broaden young people's annuity market and design more products for young people.

Now, let us give the value function.

Theorem 6. For $(t, y) \in D_{1}$, we have

$$
V(t, x, y)= \begin{cases}V_{1}(t, x)+V_{2}(t, y), & x \leq x_{1}(t, y), \\ V_{1}\left(t, x_{1}\right)+V_{2}\left(t, y+\frac{x-x_{1}}{1-k}\right), & x>x_{1}(t, y),\end{cases}
$$

and for $(t, y) \in D_{2}$, we have

$$
V(t, x, y)= \begin{cases}V_{1}(t, x)+V_{2}(t, y), & x \leq x_{0}(t), \\ V_{1}\left(t, x_{0}\right)+x-x_{0}(t)+V_{2}(t, y), & x>x_{0}(t) .\end{cases}
$$

Up till now, we have obtained the expression of the value function, the optimal investment, consumption, and annuitization strategies in terms of $V_{1}(t, x)$ and $V_{2}(t, y)$. In the next section, we will analyze the problem when the rate of buying tax-deferred annuities is restricted.

\section{The Optimal Strategies When $p_{t} \leq \bar{p}$}

The above analysis considers the optimal annuitization strategies under the assumption that buying annuities are not restricted. In fact, in order to ensure the role of Taxation and reduce the financial pressure, many countries stipulate that the number of tax-deferred annuities purchased by a person shall not exceed some point. In this section, let us consider the problem under the assumption that $p_{t} \leq \bar{p}$ for some constant $\bar{p}>0$. Using the results in Theorem 1 , we can get that the value function satisfies (14) and (15) and 


$$
\begin{aligned}
\min & \left\{\left(r+\lambda_{t}^{s}\right) V(t, x, y)-V_{t}(t, x, y)-\sup _{b_{1} \geq 0, b_{2} \geq 0}\left[\left(\widehat{r} x+(\widehat{\mu}-\widehat{r}) b_{1}+k b\right) V_{x}(t, x, y)\right.\right. \\
& \left.+\frac{\widehat{\sigma}^{2} b_{1}^{2}}{2} V_{x x}(t, x, y)+\sigma \widehat{\sigma} b_{1} b_{2} V_{x y}+\left(r y+b_{2}(\mu-r)\right) V_{y}(t, x, y)+\frac{\sigma^{2} b_{2}^{2}}{2} V_{y y}(t, x, y)\right] \\
& \left.-\sup _{0 \leq p \leq \bar{p}}\left[p V_{y}(t, x, y)-\widehat{p} V_{x}(t, x, y)\right], V_{x}(t, x, y)-1\right\}=0, \quad t<T .
\end{aligned}
$$

Thus, in this case, the optimal investment strategies are still determined by (16) and (17).
It follows from (47) that for $(1-k) V_{x}(t, x, y) \geq V_{y}(t, x, y)$, we have

$$
\begin{aligned}
& \left(r+\lambda_{t}^{s}\right) V(t, x, y) \geq \sup _{b_{1} \geq 0, b_{2} \geq 0}\left(\widehat{r} x+(\widehat{\mu}-\widehat{r}) b_{1}+k b\right) V_{x}(t, x, y)+\frac{\widehat{\sigma}^{2} b_{1}^{2}}{2} V_{x x}(t, x, y)+\sigma \widehat{\sigma} b_{1} b_{2} V_{x y} \\
& \quad+\left(r y+b_{2}(\mu-r)\right) V_{y}(t, x, y)+\frac{\sigma^{2} b_{2}^{2}}{2} V_{y y}(t, x, y)+\sup _{c \geq 0}\left[c\left(1-V_{x}(t, x, y)\right)\right]+V_{t}(t, x, y) \\
& \quad V_{x}(t, x, y) \geq 1
\end{aligned}
$$

with at least one strict equal sign. For $(1-k) V_{x}(t, x, y)<V_{y}(t, x, y)$, we have

$$
\begin{aligned}
\left(r+\lambda_{t}^{s}\right) V(t, x, y) \geq & \sup _{b_{1} \geq 0, b_{2} \geq 0}\left(\widehat{r} x+(\widehat{\mu}-\widehat{r}) b_{1}+k b\right) V_{x}(t, x, y)+\frac{\widehat{\sigma}^{2} b_{1}^{2}}{2} V_{x x}(t, x, y)+\sigma \widehat{\sigma} b_{1} b_{2} V_{x y} \\
& +\left(r y+b_{2}(\mu-r)\right) V_{y}(t, x, y)+\frac{\sigma^{2} b_{2}^{2}}{2} V_{y y}(t, x, y)+\sup _{c \geq 0}\left[c\left(1-V_{x}(t, x, y)\right)\right] \\
& +\bar{p} V_{y}(t, x, y)-(1-k) \bar{p} V_{x}(t, x, y)+V_{t}(t, x, y), \\
V_{x}(t, x, y) \geq 1 &
\end{aligned}
$$

with at least one strict equal sign.

Let $U_{1}(t, x)$ satisfy

$$
\begin{aligned}
& -\left(r+\lambda_{t}^{s}\right) U_{1}(t, x)+U_{1 t}(t, x)+\widehat{r} x U_{1 x}(t, x)+(k b-(1-k) \bar{p}) U_{1 x}(t, x)-\frac{R^{2} U_{1 x}^{2}(t, x)}{2 U_{1 x x}(t, x)}=0, \\
& U_{1}(T, x)=x, \\
& U_{1}(t, 0)=0 .
\end{aligned}
$$

Let $U_{2}(t, y)$ satisfy 


$$
\begin{aligned}
& -\left(r+\lambda_{t}^{s}\right) U_{2}(t, y)+U_{2 t}(t, y)+(r y+\bar{p}) U_{2 y}(t, y)-\frac{R^{2} U_{2 y}^{2}(t, y)}{2 U_{2 y y}(t, y)}=0, \\
& U_{2}(T, y)=g(y), \quad y \geq 0, \\
& U_{2}(t, 0)=V_{1}\left(t, x_{1}(t, y)\right)-U_{1}\left(t, x_{1}(t, y)\right), \quad t \geq 0 .
\end{aligned}
$$

Define $\tilde{x}_{0}(t)$ by

$$
U_{1 x}\left(t, \tilde{x}_{0}(t)\right)=1
$$

Appendix B gives us numerical solutions of $U_{1}(t, x)$ and $U_{2}(t, y)$. Figures 3 and 4 give us the following theorem.

Theorem 7. $U_{1}(t, x)$ is a concave increasing positive function with respect to $x$, and $U_{1 x}(t, x)$ is increasing with respect to $t$ and

$$
U_{1 x}(t, x)>V_{1 x}(t, x), \quad x \geq 0 .
$$

According to Theorem 7, we can obtain that

$$
V_{1}^{\prime}\left(t, \tilde{x}_{0}\right)<U_{1}^{\prime}\left(t, \tilde{x}_{0}\right)=1=V_{1}^{\prime}\left(t, x_{0}(t)\right)
$$

Then, we can get

$$
\tilde{x}_{0}(t)>x_{0}(t), \quad \forall t \geq 0 .
$$

Next, we consider the form of the value function in $D_{1}$ and $D_{2}$, respectively.

For $t \in D_{1}$, define

$$
\begin{aligned}
w(t, x, y) & = \begin{cases}V_{1}(t, x)+V_{2}(t, y), & x \leq x_{1}(t, y), \\
U_{1}(t, x)+U_{2}(t, y), & x_{1}(t, y)<x<\tilde{x}_{0}(t), \\
U_{1}\left(t, \tilde{x}_{0}(t)\right)+x-\tilde{x}_{0}(t)+U_{2}(t, y), & x \geq \tilde{x}_{0}(t),\end{cases} \\
\Delta_{1}^{*}(t, x, y) & = \begin{cases}0, & x \leq \tilde{x}_{0}(t), \\
x-\tilde{x}_{0}(t), & x>\tilde{x}_{0}(t),\end{cases} \\
p^{*}(t, x, y) & = \begin{cases}0, & x \leq x_{1}(t, y), \\
\bar{p}, & x>x_{1}(t, y) .\end{cases}
\end{aligned}
$$

For $t \in D_{2}$, define

$$
\begin{aligned}
w(t, x, y) & = \begin{cases}V_{1}(t, x)+V_{2}(t, y), & x \leq x_{0}(t), \\
V_{1}\left(t, x_{0}(t)\right)+x-x_{0}(t)+V_{2}(t, y), & x>x_{0}(t),\end{cases} \\
\Delta_{1}^{*}(t, x, y) & = \begin{cases}0, & x \leq x_{0}(t), \\
x-x_{0}(t), & x>x_{0}(t),\end{cases} \\
p^{*}(t, x, y) & =0 .
\end{aligned}
$$

Then, we have the following corollary.

Lemma 1. $w(t, x, y)$ defined above satisfies (14), (15), and (47).
Proof. According to (20), (21), (51), (52), (54), and (55), we can get that $w(t, x, y)$ satisfies (14) and (15).

For $t \in D_{1}, x \leq x_{1}(t, y)$, we have that

$$
\begin{aligned}
(1-k) w_{x}(t, x, y) & =(1-k) V_{1}^{\prime}(t, x) \geq(1-k) V_{1}^{\prime}\left(t, x_{1}(t, y)\right)=V_{2}^{\prime}(t, y)=w_{y}(t, x, y) \\
w_{x}(t, x, y) & =V_{1}^{\prime}(t, x)>1
\end{aligned}
$$

Thus, we can get that 


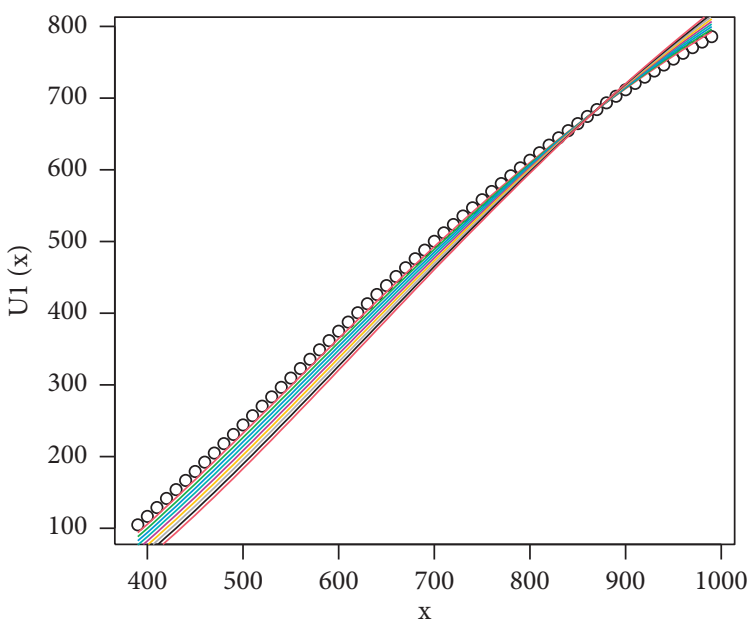

Figure 3: $U_{1}(t, x)$

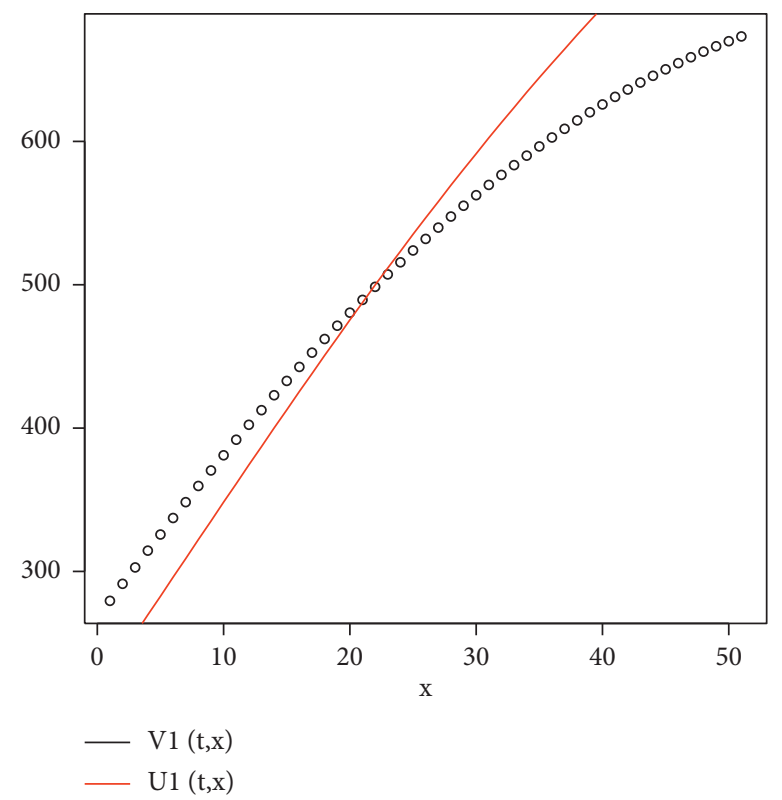

Figure 4: A comparison of $V_{1}(t, x)$ and $U_{1}(t, x)$

$$
\begin{aligned}
c_{t}^{*} & =0, \\
p_{t}^{*} & =0 .
\end{aligned}
$$

Since $V_{1}(t, x)$ satisfies (23), and $V_{2}(t, y)$ satisfies (24), it follows immediately that $w(t, x, y)$ satisfies (47).

For $t \in D_{1}, x_{1}(t, y)<x<\tilde{x}_{0}(t)$, we have

$$
\begin{aligned}
w_{x}(t, x, y) & =U_{1}^{\prime}(t, x)>1, \\
(1-k) w_{x}(t, x, y) & =(1-k) U_{1}^{\prime}(t, x) \leq(1-k) V_{1}^{\prime}(t, x) \leq(1-k) V_{1}^{\prime}\left(t, x_{1}(t, y)\right), \\
(1-k) V_{1}^{\prime}\left(t, x_{1}(t, y)\right) & =V_{2}^{\prime}(t, y)=U_{2}^{\prime}(t, y)=w_{y}(t, x, y) .
\end{aligned}
$$


It implies that

$$
\begin{aligned}
& c_{t}^{*}=0, \\
& p_{t}^{*}=\bar{p} .
\end{aligned}
$$

Since $U_{1}(t, x)$ satisfies (50), and $U_{2}(t, y)$ satisfies (53), it follows immediately that $w(t, x, y)$ satisfies (47).

For $t \in D_{1}, x \geq \tilde{x}_{0}(t)$, we have

$$
\begin{aligned}
(1-k) w_{x}(t, x, y) & =(1-k) U_{1}^{\prime}\left(t, \tilde{x}_{0}(t)\right)=(1-k) V_{1}^{\prime}\left(t, x_{0}(t)\right)<(1-k) V_{1}^{\prime}\left(t, x_{1}(t, y)\right) \\
(1-k) V_{1}^{\prime}\left(t, x_{1}(t, y)\right) & =V_{2}^{\prime}(t, y)=U_{2}^{\prime}(t, y)=w_{y}(t, x, y), \\
w_{x}(t, x, y) & =1 .
\end{aligned}
$$

Thus, we can get $p_{t}^{*}=\bar{p}$. Clearly, $w(t, x, y)$ is the solution of (47).

The cases for $(t, y) \in D_{2}$ can be proved with a similar analysis, and we can obtain that $w(t, x, y)$ satisfies (47) finally.

The concavity of $V_{1}(t, x), U_{1}(t, x), \quad V_{2}(t, y)$, and $U_{2}(t, y)$ indicates that $w(t, x, y)$ satisfies the polynomial growth condition. So, using Theorem 3.5.2 in [13], we can immediately get that $w(t, x, y)=V(t, x, y)$.

Remark 4. Obviously, the boundary of buying annuities in this section is the same as the one in Section 3. So, the suggestions proposed in Section 3 can also apply when the rate of buying annuities is restricted.

Remark 5. The form of $p^{*}$ indicates that properly raising the upper bound $\bar{p}$ can increase the rate of buying annuities.

\section{Appendix}

Owing to the existence of tax and the time dependence of the value function, we cannot get explicit solutions of the differential equations satisfied by $V_{1}(t, x), V_{2}(t, y)$ and $U_{1}(t, x), U_{2}(t, y)$. So, numerical methods should be applied. One of the generally used numerical methods is called the standard finite difference method. The steps of the method are as follows:

(i) Gridding the domain of the unknown function.

(ii) Replacing derivative functions in the differential equation with difference functions expressed by the function values of the grid points.

(ii) Using the boundary conditions and solving the difference equations to deduce the function values of the grid points.

According to [15], we know that as long as the solution of the differential equation is growing linearly, the method is stable. Thus, we use this method to get the value function of our problem.

In this paper, we suppose that the investor is 22 years old at time 0 , and by using the life table data of China Life Insurance and doing regression analysis, we can approximate the hazard rate of the individual at time $t$ with $\lambda_{t}^{s}=0.001+0.0006 t^{2}$.

\section{A. The solutions of $V_{1}(t, x)$ and $V_{2}(t, y)$}

In order to get $V_{1}(t, x)$, let us discuss the numerical solution of the partial differential equation

$$
\begin{aligned}
\left(r+\lambda_{t}^{s}\right) V_{1}(t, x)-\widehat{r} x V_{1 x}(t, x)-k b V_{1 x}(t, x)+\frac{R^{2} V_{1 x}^{2}(t, x)}{2 V_{1 x x}(t, x)} & =V_{1 t}(t, x) \\
V_{1}(t, 0) & =0
\end{aligned}
$$

Clearly, the definition domain of $V_{1}(t, x)$ is $[0, T] \times \mathscr{R}^{+} \cup 0$. Fixing $h>0$, denoting $a_{i j}=V_{1}((i-1) h$, $(j-1) h)$, and letting $m-1=T / h, n \in \mathbb{Z}^{+}$, we construct an $m \times n$ matrix $A$ :

$$
\left(\begin{array}{ccccc}
a_{11} & a_{12} & a_{13} & \cdots & a_{1 n} \\
a_{21} & a_{22} & a_{23} & \cdots & a_{2 n} \\
\cdot & \cdot & \cdot & \cdot & \cdot \\
\cdot & \cdot & \cdot & \cdot & \cdot \\
\cdot & \cdot & \cdot & \cdot & \cdot \\
a_{m 1} & a_{m 2} & a_{m 3} & \cdots & a_{m n}
\end{array}\right) .
$$

Obviously, the matrix is the numerical solution of $V_{1}(t, x)$ when $h \longrightarrow 0$.

Since $V_{1}(t, 0)=0$, we can get the first column of $A$. That is,

$$
a_{i 1}=0, \quad i=1,2, \ldots, m
$$

Furthermore, for an initial wealth $x=0$, we know that the investment strategy is $b_{1}=0$, that is,

$$
\frac{V_{1 x}^{2}(t, 0)}{V_{1 x x}(t, 0)}=0 \text {. }
$$

Then, for $h \longrightarrow 0$, we have 


$$
\frac{V_{1 x}^{2}(t, h)}{V_{1 x x}(t, h)}=0
$$

It means that

$$
\begin{aligned}
& \left(r+\lambda_{t}^{s}\right) V_{1}(t, h)-\widehat{r} x V_{1 x}(t, h)-k b V_{1 x}(t, h) \\
& =V_{1 t}(t, h), \quad h \longrightarrow 0 .
\end{aligned}
$$

Using the finite difference method, we can substitute $V_{1 x}(t, h)$ with $\left(\left(V_{1}(t, h)-V_{1}(t, 0)\right) / h\right)$ and get the following differential equation with respect to $t$

$$
\left(r+\lambda_{t}^{s}\right) V_{1}(t, h)=V_{1 t}+(\widehat{r} h+k b) \frac{V_{1}(t, h)-V_{1}(t, 0)}{h} .
$$

By doing some simple calculations, we can obtain that

$$
V_{1}(t, h)=V_{1}(0, h) e^{\int_{0}^{t} L(s) \mathrm{d} s},
$$

where $L(s)=\lambda_{s}^{s}+r-\widehat{r}-(k b / h)$.

According to $(20)$, we know that $V_{1}(T, h)=h$. Combining with (A.8), we can get that

$$
V_{1}(0, h)=h e^{-\int_{0}^{T} L(s) \mathrm{d} s} .
$$

Then, we obtain the second column of $A$.

In order to get the first two rows of $A$, we consider the pair $\left(a_{13}, a_{23}\right)$ first. Substituting $V_{1 x}(0,2 h), V_{1 t}$ and $\left(V_{1 x}^{2}\right.$ $\left.(0,2 h) / V_{1 x x}(0,2 h)\right)$ with $\left(\left(a_{13}-a_{12}\right) / h\right),\left(\left(a_{23}-a_{13}\right) / h\right)$ and $\left(\left(a_{13}-a_{12}\right)^{2} /\left(a_{13}+a_{11}-2 a_{12}\right)\right)$, respectively, we can get

$$
\left(r+\lambda_{0}^{s}\right) a_{13}=\frac{a_{23}-a_{13}}{h}+(\widehat{r} h+k b) \frac{a_{13}-a_{12}}{h}-\frac{R^{2}\left(a_{13}-a_{12}\right)^{2}}{2\left(a_{13}+a_{11}-2 a_{12}\right)} .
$$

Similarly, we can also get

$$
\left(r+\lambda_{h}^{s}\right) a_{23}=\frac{a_{23}-a_{13}}{h}+(\hat{r} h+k b) \frac{a_{23}-a_{22}}{h}-\frac{R^{2}\left(a_{23}-a_{22}\right)^{2}}{2\left(a_{23}+a_{21}-2 a_{22}\right)} .
$$

Combining with the two equations, we can get $a_{13}$ and $a_{23}$.

With the same method, we can also get $a_{14}, a_{24}, a_{15}, a_{25}, \ldots$. Then, the first two rows of $A$ are obtained. At last, we can deduce the left elements from left to right, from top to bottom by solving the corresponding difference equations.

Next, let us consider $V_{2}(t, y)$. We have to find the numerical solution of the partial differential equation

$$
\begin{aligned}
& \left(r+\lambda_{t}^{s}\right) V_{2}(t, y)-r y V_{2 y}(t, y)+\frac{R^{2} V_{1 y}^{2}(t, y)}{2 V_{2 y y}(t, y)}=V_{2 t}(t, y), \\
& V_{2}(t, y)=0 \\
& V_{2}(T, y)=g(y) .
\end{aligned}
$$

Similarly, we construct an $m \times n$ matrix $B$ :

$$
\left(\begin{array}{ccccc}
b_{11} & b_{12} & b_{13} & \cdots & b_{1 n} \\
b_{21} & b_{22} & b_{23} & \cdots & b_{2 n} \\
\cdot & \cdot & \cdot & \cdot & \cdot \\
\cdot & \cdot & \cdot & \cdot & \cdot \\
\cdot & \cdot & \cdot & \cdot & \cdot \\
b_{m 1} & b_{m 2} & b_{m 3} & \cdots & b_{m n}
\end{array}\right),
$$

where $b_{i j}=V_{2}((i-1) h,(j-1) h)$ and $m-1=(T / h), n$ $\in \mathbb{Z}^{+}$. Using the boundary conditions, we can get the first column and the last row of $B$. That is,

$$
\begin{aligned}
b_{i 1} & =0, \quad i=1,2, \ldots, m, \\
b_{m j} & =g((j-1) h), \quad j=1,2, \ldots, n .
\end{aligned}
$$

What is more, for $h \longrightarrow 0$, we have

$$
\frac{V_{2 y}^{2}(t, h)}{V_{2 y y}(t, h)}=0 \text {. }
$$

Thus, we can get

$$
\left(r+\lambda_{t}^{s}\right) V_{2}(t, h)-r y V_{2 y}(t, h)=V_{2 t}(t, h), \quad h \longrightarrow 0 .
$$

Using the finite difference method, we can substitute $V_{2 y}(t, h)$ with $\left(\left(V_{2}(t, h)-V_{2}(t, 0)\right) / h\right)$ and get the following differential equation with respect to $t$ :

$$
\left(r+\lambda_{t}^{s}\right) V_{2}(t, h)=V_{2 t}+r h \frac{V_{2}(t, h)-V_{2}(t, 0)}{h} .
$$

By doing some simple calculations, we can obtain that

$$
V_{2}(t, h)=V_{2}(T, h) e^{-\int_{t}^{T} \lambda_{s}^{s} \mathrm{~d} s}
$$

Then, we get the second column of $B$. Since the first two columns and the last row of $B$ are obtained, left to right, bottom to top, we can get the elements left.

The R program of solving $V_{1}(t, x)$ and $V_{2}(t, y)$ and the $\mathrm{R}$ program of plotting Figures 1 and 2 are given in the file A.R in the attachment. Clearly, the image of $V_{1}(t, x)$ with respect to $x$ for different $t$ illustrates that $V_{1}(t, x)$ is a concave increasing positive function with respect to $x$. Similarly, $V_{2}(t, y)$ is a concave increasing positive function with respect to $y$.

\section{B. The solutions of $U_{1}(t, x), U_{2}(t, y)$ and a comparison of $U_{1}(t, x)$ and $V_{1}(t, x)$}

Since $U_{1}(t, x), U_{2}(t, y)$ satisfy the same forms of differential equations as $V_{1}(t, x), V_{2}(t, y)$, respectively, the processes of solving $U_{1}(t, x), U_{2}(t, y)$ are the same as the processes of solving $V_{1}(t, x), V_{2}(t, y)$. The $\mathrm{R}$ program of solving $U_{1}(t, x), U_{2}(t, y)$ and the R program of plotting Figures 3-5 are presented in the file B.R in the attachment. 


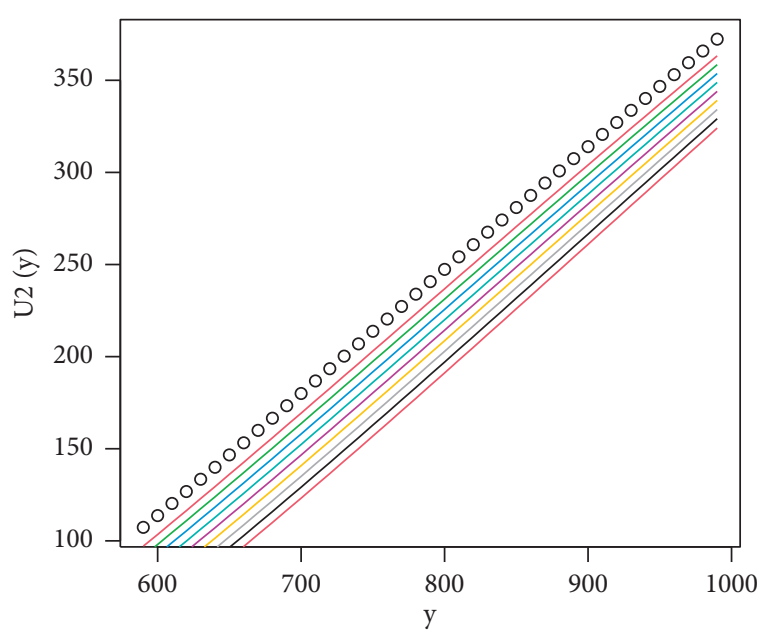

Figure 5: $U_{2}(t, y)$

It is shown from Figure 3 that $U_{1}(t, x)$ is a concave increasing positive function with respect to $x$. What is more, Figure 4 tells us that

$$
\begin{gathered}
U_{1}(t, x) \leq V_{1}(t, x), \\
U_{1 x}(t, x) \leq V_{1 x}(t, x) .
\end{gathered}
$$

\section{Data Availability}

No data were used in this paper.

\section{Conflicts of Interest}

The author declares no conflicts of interest.

\section{Acknowledgments}

This work was supported by National Natural Science Foundation of China (11901404).

\section{References}

[1] Y. Wang, The Development of Security and Whole Care System for the Aged in China, Springer, Berlin, Germany, 2018.

[2] M. A. Milevsky and C. Robinson, "Self-annuitization and ruin in retirement," North American Actuarial Journal, vol. 4, no. 4, pp. 112-124, 2000.

[3] M. A. Milevsky and V. R. Young, "Annuitization and asset allocation," Journal of Economic Dynamics and Control, vol. 31, no. 9, pp. 3138-3177, 2007.

[4] R. Gerrard, B. Højgaard, and E. Vigna, "Choosing the optimal annuitization time post-retirement," Quantitative Finance, vol. 12, no. 7, pp. 1143-1159, 2012.

[5] T. Wang and V. R. Young, "Maximizing the utility of consumption with commutable life annuities," Insurance: Mathematics and Economics, vol. 51, no. 2, pp. 352-369, 2012.

[6] D. Hainaut and G. Deelstra, "Optimal timing for annuitization, based on jump diffusion fund and stochastic mortality," Journal of Economic Dynamics and Control, vol. 44, no. 1, pp. 24-146, 2014.
[7] T. Wang and V. R. Young, "Optimal commutable annuities to minimize the probability of lifetime ruin," Insurance: Mathematics and Economics, vol. 50, no. 1, pp. 200-216, 2012.

[8] B. Temocin, R. Korn, and S. Kestel, "Constant proportion portfolio insurance in defined contribution pension plan management under discrete-time trading," Annals of Operations Research, vol. 260, no. 3, pp. 1-30, 2018.

[9] M. Xu, M. A. Sherris, and S. Wenqiang, "Portfolio insurance strategies for a target annuitisation fund," Astin Bulletin, vol. 1, p. 1, 2020.

[10] R. M. Dammon, C. S. Spatt, and H. H. Zhang, "Optimal asset location and allocation with taxable and tax-deferred investing," The Journal of Finance, vol. 59, no. 3, pp. 999-1037, 2004.

[11] T. Jalbert, E. Rask, and M. Jalbert, "Optimal tax deferral choices in the presence of changing tax regimes," Social science electronic publishing, vol. 23, no. 1, 2011.

[12] T. Jalbert, J. Clayton, and E. Rask, "The effect of credits on optimal tax-deferral strategie," Financial Services Review, vol. 16, pp. 41-54, 2007.

[13] H. Pham, Continuous-Time Stochastic Control and Optimization with Financial Applications, Springer-Verlag, Berlin, Germany, 2009.

[14] J. Gao, "Optimal investment strategy for annuity contracts under the constant elasticity of variance (CEV) model," Insurance: Mathematics and Economics, vol. 45, no. 1, pp. 9-18, 2009.

[15] S. Rucker, "Exact finite difference scheme for an advectionreaction equation," Journal of Difference Equations and Applications, vol. 9, no. 11, pp. 1007-1013, 2003. 